Conclusions: Nitrofurantoin and ciprofloxacin remain viable alternatives, respectively for uncomplicated and complicated UTI. Trends of antimicrobial resistance should be monitored at the local level as important changes occur over time.

1011

\section{EMPLOYMENT CHARACTERISTICS OF PEDIATRIC NURSE PRACTITIONER GRADUATES IN THE USA: AN ADDITIONAL WORKFORCE FOR THE HEALTH CARE OF CHILDREN}

\author{
P.J. Allen \\ School of Nursing, Yale University, \\ New Haven, CT, USA
}

Aims: Describe the characteristics of employment, children cared for, frequency of role functions by practice setting (primary care, specialty practice), and interest in doctoral education of recent PNP graduates.

Method: A 92-item multiple choice and short answer survey tool was sent to graduates from a convenience sample of universities.

Results: The surveys were sent to over 280 graduates with a return rate of $53 \%$ (range $28 \%$ $86 \%$ by university). Of those responding $95 \%$ were employed with $80 \%$ employed full-time. Thirty-two percent were employed in primary care settings with an additional $10 \%$ in school-based clinics. Fiftythree percent were employed in specialty practice in either inpatient or outpatient settings. They often cared for children with acute conditions $(60 \%)$ and chronic health conditions (53\%) irrespective of clinical setting.

Role functions varied by practice setting with provision of preventive health interventions, growth and development counseling, lifestyle behavior counseling, and initiation of referrals being performed more frequently $(p<.05)$ by PNPs in primary care or school-based settings. Provision of case management, staff development, policy and program development, writing for publication, participation in research, system change activities, delegation of care and supervision of students was performed more often $(p<.05)$ by PNPs in hospital settings.

Forty-six percent indicated they were interested in doctoral education with $22 \%$ indicating interest in a
$\mathrm{PhD}$ and $78 \%$ indicating interest in the Doctorate of Nursing Practice.

Conclusion: Pediatric Nurse Practitioners are highly educated and skilled additional pediatric health care providers and should be utilized to their fullest potential.

\section{2}

\section{GOOD NURSING TRAITS: A COMPARISON OF CHILDREN'S VIEWS}

\author{
P. Ferri' ${ }^{1}$, V.L. Napoli' ${ }^{1}$, F. Cerullo \\ ${ }^{1}$ University of Modena and Reggio Emilia, ${ }^{2}$ Hospital \\ of Modena, Modena, Italy
}

Background: Excepting Randall's study (2008), literature about children's feedback of nursing is poor and remarkably dated (Fleitas 1997, Bluebond-Langner 1978). Yet, interviewed children gave a very detailed account, which resulted deeply different from their parents' or care assistants' reports (Miller 2000, Scott 2000).Goal of the study: We interviewed hospitalized children asking for the traits of good nursing. This qualitative study has a phenomenological approach.

Methods: A qualitative interview was used to collect data: we interviewed 10 children admitted at Pediatria of the Azienda Ospedaliera-Universitaria Policlinico in Modena (Italy) in early 2009. Children were not selected according to probabilistic methods. Research was authorized by Unit Direction; we ensured anonymity to parents who gave written consent. The interviewer introduced himself to children explaining the aims of the research and asking them if they were in the mood for answering.

Findings/results: Outcomes may be divided into 4 groups:

1. Natural-born qualities"A good nurse should be a special person in order to help children"

2. Learning from aptitude"A good nurse should be cheerful with children who are afraid of cures"

3. Learning from experience"A good nurse should know and then remember: he should be experienced with children"

4. Cognitive and psycho-physical learning"A good nurse should be qualified, I like nurses who explain what they are doing" 